\title{
前列腺素类化合物的高效不对称合成新方法
}

徐明华

南方科技大学化学系/深圳格拉布斯研究院, 深圳 518055

E-mail: xumh@sustech.edu.cn

\section{A new efficient method for asymmetric synthesis of prostaglandins}

\author{
Ming-Hua Xu \\ Department of Chemistry and Shenzhen Grubbs Institute, Southern University of Science and Technology, Shenzhen 518055, China \\ E-mail: xumh@sustech.edu.cn
}

doi: 10.1360/TB-2021-0497

前列腺素(prostaglandin, $\mathrm{PG}$ )是一类广泛存在于体内的自 体活性物质, 介导体内多种生理功能. 1934年, von Euler ${ }^{[1]}$ 首 次发现了前列腺素, 当时认为此物质可能是由前列腺所分泌, 因此命名为前列腺素. 1957年Bergström与Samuelsson ${ }^{[2]}$ 分离 出两种前列腺素纯品 $\left(\mathrm{PGF}_{1}\right.$ 和 $\left.\mathrm{PGF}_{2 \alpha}\right)$, 并在后续研究中阐明 了前列腺素的结构和代谢过程. 20 世纪60年代出现前列腺素 药物的研究高潮. 1982年, Sune Bergström、Bengt Samuelsson 和John Vane三位科学家因在前列腺素及有关生物活性物质 的研究方面的卓越贡献共同获得了诺贝尔生理学或医学奖.

前列腺素在体内由花生四烯酸所合成, 结构为由一个五 元环和两条侧链构成的不饱和脂肪酸，对内分泌、生殖、消 化、血液、呼吸、心血管、泌尿和神经系统均有作用. 目前, 在世界范围内有超过 20 种前列腺素类上市药物. 由于前列腺 素类化合物独特的结构和广泛的生物活性, 发展前列腺素的 高效合成方法具有非常重要的意义，在过去的50多年间，前 列腺素一直是合成化学家的重要目标分子. $\mathrm{PGF}_{2 \alpha}$ 是前列腺 素家族中最复杂的化合物，其核心结构为具有四个连续手性 中心的环戊烷骨架和两个侧链(图1(a)).1964年, Corey等人 ${ }^{[3]}$ 首次实现了 $\mathrm{PGF}_{2 a}$ 的全合成, 随后, 众多著名的有机化学家如 Woodward、Stork、Noyori、Danishefsky、Aggarwal、Baran、Grubbs等也在前列腺素的合成方面做出了许多重要的研 究工作. 尽管前列腺素的合成取得了重要进展, 已经开发了 多条合成路线，但是，目前前列腺素类药物的工业合成仍然 主要依赖Corey内酯(10). Corey内酯可以从环戊二烯9出发经 过9步合成得到，从Corey内酯出发仍需要多步反应才能够得 到前列腺素，有些前列腺素分子甚至需要超过10步来合成 (图1(b)). 南方科技大学张绪穆、陈根强研究团队 ${ }^{[4]}$ 在前列腺 素的合成研究中取得了突破性进展，开发了一种全新的简
洁、高效合成方法, 以简单易得的 $\beta$-酩酰胺 11 为原料, 经 4 步 反应可以构建关键中间体 15 , 再仅需 2 步转化就可以高效合 成 $\mathrm{PGF}_{2 \alpha}(\mathbf{1})$, 该成果于 2021 年 5 月 27 日在线发表于Nature Chemistry.

前列腺素不对称合成中的主要难点在于其核心环戊烷 骨架中四个连续手性中心的精准构建以及两个侧链的高效 引人. 2000 年, 张绪穆课题组 ${ }^{[5,6]}$ 报道了首例铑催化的 1,6 - 烯炔 的环异构化反应，可以实现手性五元环化合物的高效构建， 对于大多数底物, 采用常见的 $2,2^{\prime}$-双-(二苯膦基)-1, $1^{\prime}$-联芸 (BINAP)作为手性配体, 该反应就可以获得非常高的对映选 择性.

他们设想以铑催化的不对称烯炔环异构化反应为关键 步骤构建手性环伐烷骨架, 进而实现前列腺素 $\mathrm{PGF}_{2 \alpha}$ 的不对 称合成. 对前列腺素 $\mathrm{PGF}_{2 \alpha}$ 进行了逆合成分析(图1(c)), 发现 目标化合物 $\mathrm{PGF}_{2 \alpha}(\mathbf{1})$ 可以通过关键中间体 15 , 经过烯烃交叉 复分解反应和Wittig反应合成得到，而关键中间体 15 则可以 通过1,6-烯炔底物 13 的不对称环异构化反应再经还原得到, 1,6-烯炔底物 13 可以通过化合物 12 和化合物 18 的锂盐加成反 应合成得到，化合物 12 则可以通过简单易得的 $\beta$-酮酰胺 11 的 不对称氢化和现场硅基保护得到.

基于上述逆合成分析, 他们以 $\beta$-酮酰胺 11 为起始原料, 对 前列腺素 $\mathrm{PGF}_{2 \alpha}$ 的合成进行了探索(图2). 采用张绪穆课题 组 ${ }^{[7]}$ 前期自主开发的 $\mathrm{f}-\mathrm{amphox}(\mathbf{L} \mathbf{1})$ 为手性配体, $\beta$-酮酰胺 11在 铱催化下羰基被对映选择性还原并以 $70 \%$ 的收率和 $94 \%$ ee得 到化合物 12, 12 中的Weinreb酰胺在锂试剂作用下可以顺利转 化为 $1,6-$ 烯炔化合物 13. 以铑为催化剂以及 $(S)$-BINAP为手性 配体, 1,6-烯炔化合物 $\mathbf{1 3}$ 发生不对称烯炔环异构化反应, 能够 以 $85 \%$ 的收率、 $98 \%$ 的对映选择性和 $>20 / 1$ 的非对映选择性 
(a) Representative examples of PGs and related drugs<smiles>CCCCC[C@H](O)C/C=C/[C@H]1[C@@H](O)C[C@H](O)[C@H]1C/C=C/CCCC(=O)O</smiles><smiles>CCCCCCC(O)C=CC1[C@H](O)CC(=O)[C@@H]1CC=CCCCC(=O)O</smiles>
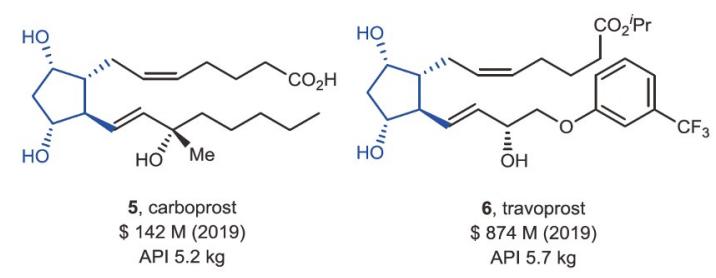

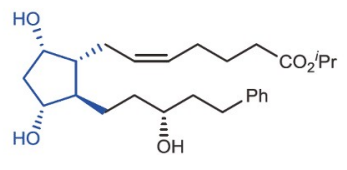

3, latanoprost \$, latanoprost API $15 \mathrm{~kg}$

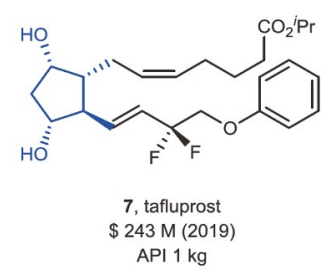<smiles>CCNC(=O)CCC/C=C/C[C@@H]1C(/C=C/[C@H](O)CCc2ccccc2CC)[C@H](O)C[C@H]1O</smiles>

4, bimatoprost

\$ 1256 M (2019) API $38 \mathrm{~kg}$

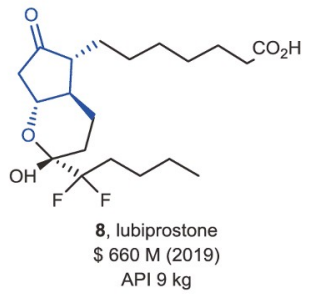

(b) Corey's synthesis of PGF $2 \alpha$
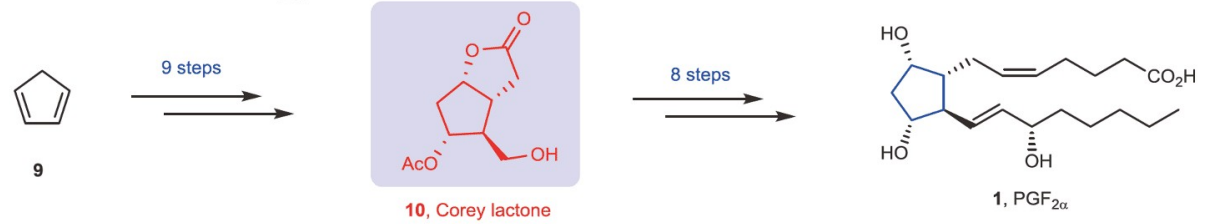

(c) Retrosynthetic analysis of $\mathrm{PGF}_{2 \alpha}$
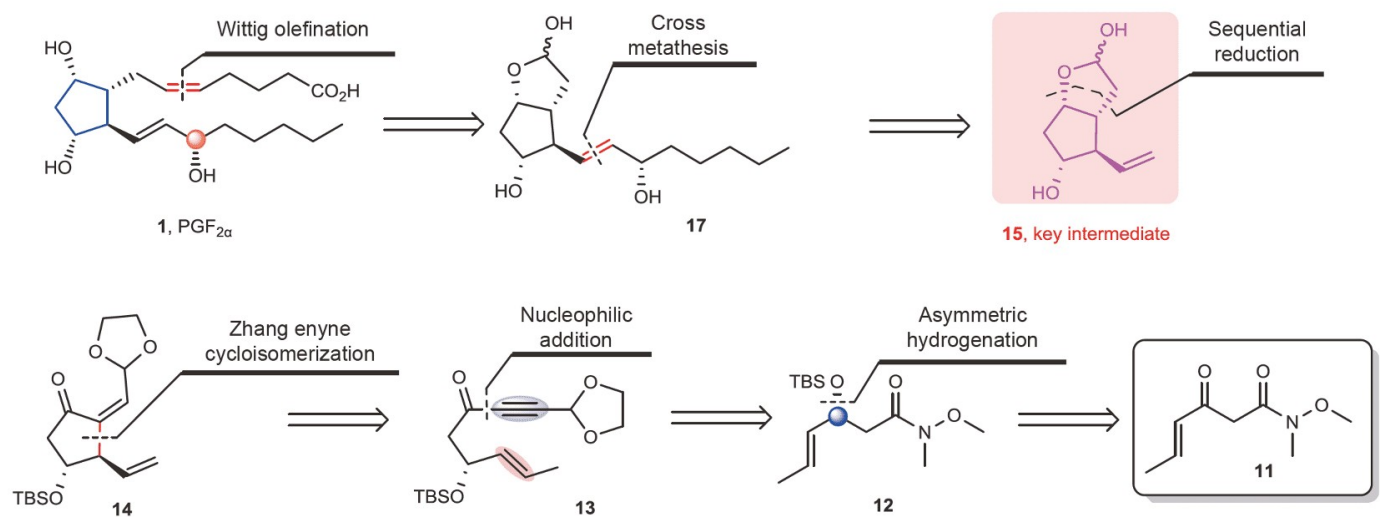

图 1 (网络版彩色)前列腺素的结构及其合成方法

Figure 1 (Color online) Structure of prostaglandins and their synthetic methods

得到化合物 14. 化合物14经过还原后，通过后处理方式的改 变可以选择性地得到关键中间体 15 或 16.

关键中间体 15在Hoveyda-Grubbs二代催化剂的作用下可 以和手性醇中间体 19 发生交叉复分解反应顺利得到化合物 17 , 化合物 17 再经过一步经典的Wittig反应就可以 $15 \%$ 的总收 率顺利得到前列腺素 $\mathrm{PGF}_{2 \alpha}(\mathbf{1})$. 采用类似的合成路线, 拉坦前 列素(latanoprost，3)、卡波前列素(carboprost，5)和氯前列烯 醇( cloprostenol，20)也能够分别以 $5.7 \% 、 23 \%$ 以及 $19 \%$ 的总 收率合成得到(图3(a)). 值得一提的是, fluprostenol(21)可以 以 $27 \%$ 的总收率和 $23 \mathrm{~g}$ 的规模合成得到(图3(b)).

张绪穆、陈根强研究团队设计了不同于Corey内酯的新 的手性环戊烷中间体，发展了一个可以简洁、高效合成前列 腺素类化合物的新方法. 从简单易得的 $\beta$-酮酰胺出发, 以他们 课题组自主发展的铱催化的不对称酮还原和铑催化的不对 称烯炔环异构化为关键步骤，仅4步反应就可以实现手性环 戊烷中间体 15 和 16 的高效高选择性构建，再经两步反应就可 
<smiles>C#CC1OCCO1</smiles>

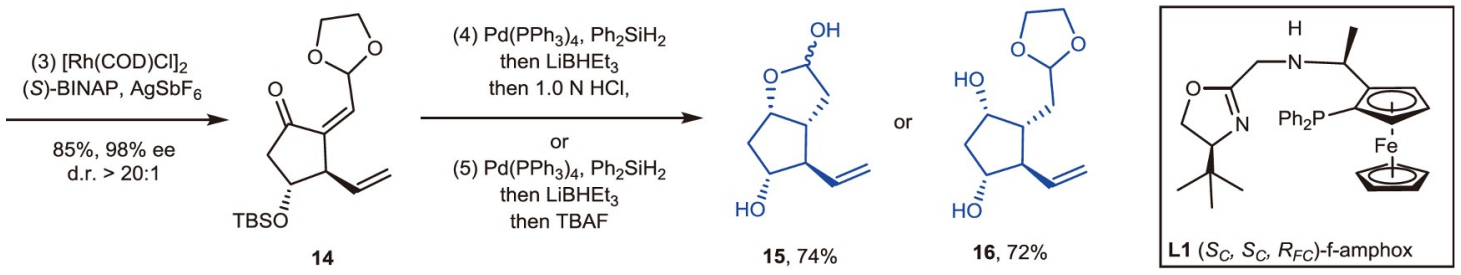

图 2 (网络版彩色)前列腺素关键中间体15和16的高效不对称合成

Figure 2 (Color online) Efficient asymmetric synthesis of two key intermediates $\mathbf{1 5}$ and $\mathbf{1 6}$

(a) Completion of the total synthesis of $\mathrm{PGF}_{2 \alpha^{\prime}}$ latanoprost, carboprost, and cloprostenol
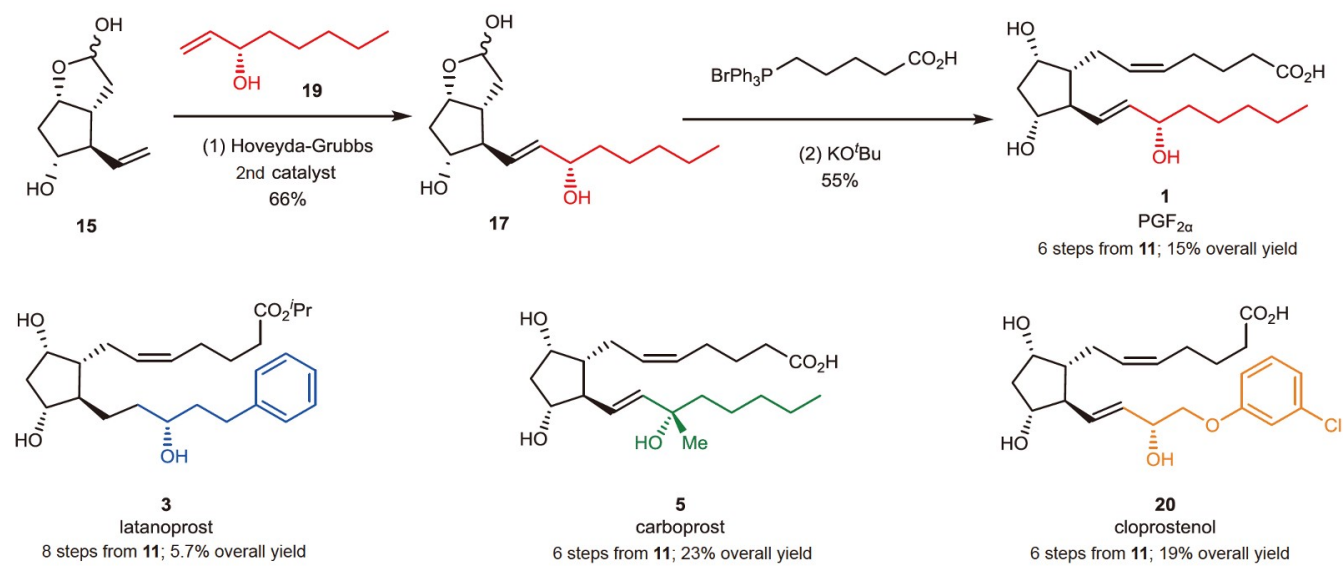

(b) Total synthesis of fluprostenol and travoprost

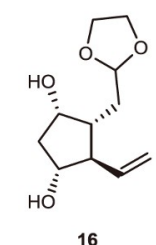

16
23 g prepared

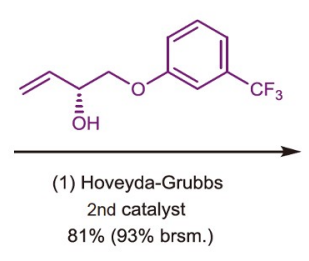

$81 \%(93 \%$ brsm.)<smiles>O[C@H](/C=C/[C@H]1O[C@@H](CC2OCCO2)[C@H](O)C[C@H]1O)COc1cccc(C(F)(F)F)c1</smiles>

$26 \mathrm{~g}$ prepared
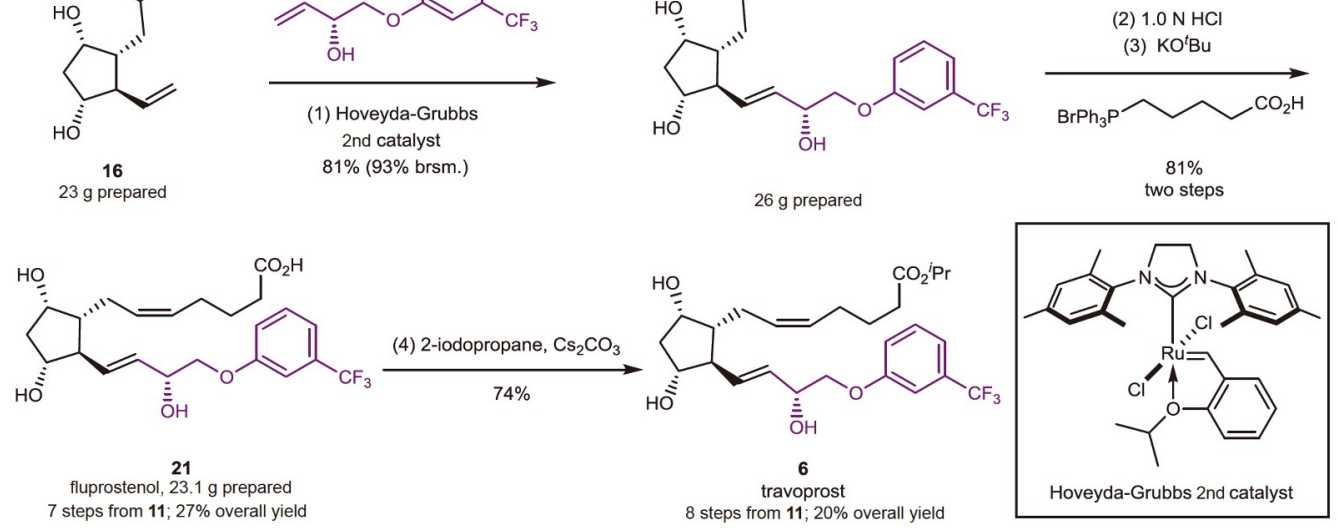

图 3 (网络版彩色)前列腺素类化合物的最终合成

Figure 3 (Color online) Completion of the total synthesis of prostaglandins 
以合成得到一系列前列腺素化合物; 而且通过关键中间体16，该研究极大地提高了前列腺素化合物的合成效率，对前列腺 能够以大于 $20 \mathrm{~g}$ 的规模合成得到氟前列醇(fluoprostenol, 21). 素药物研究具有重要意义.

\section{参考文献}

1 v. Euler U S. Information of the pharmacological effects from native secretion and extracts of male accessorygender anderoid glands. Arch Exp Pathol Pharmakol, 1934, 175: 78-84

2 Bergström S, Sjovall J. The isolation of prostaglandin. Acta Chem Scand, 1957, 11: 1086

3 Corey E J, Weinshenker N M, Schaaf T K, et al. Stereo-controlled synthesis of dl-prostaglandins $\mathrm{F}_{2 \alpha}$ and $\mathrm{E}_{2}$. J Am Chem Soc, 1969, 91 : 5675-5677

4 Zhang F, Zeng J, Gao M G, et al. Concise, scalable and enantioselective total synthesis of prostaglandins. Nat Chem, 2021, 13: 692-697

5 Cao P, Wang B, Zhang X. Rh-catalyzed enyne cycloisomerization. J Am Chem Soc, 2000, 122: 6490-6491

6 Cao P, Zhang X. The first highly enantioselective Rh-catalyzed enyne cycloisomerization. Angew Chem Int Ed, 2000, 39: 4104-4106

7 Wu W, Liu S, Duan M, et al. Iridium catalysts with f-amphox ligands: Asymmetric hydrogenation of simple ketones. Org Lett, 2016, 18: 2938-2941 